\title{
Discharge of psychiatric patients against psychiatrist's advice
}

\author{
Shiva Soraya ${ }^{1,2}$, Mohaddese Zolfi $^{1} * \mathbb{C}$, Ruohollah Seddigh ${ }^{1,2}$, Homa Mohammadsadeghi $^{1,2}$, Fatemeh Hadi ${ }^{1,2}$ \\ Received: 17 Feb 2020 \\ Published: 19 Jul 2021
}

\begin{abstract}
Background: Discharge from the hospital against the doctor's advice and refusal of receiving treatment is one of the significant issues at the time of hospitalization, which is especially crucial in relation to psychiatric patients. It can exacerbate the disorder and the subsequent complications and increase further hospital admissions. The present study was designed to evaluate the causes of discharge from the hospital and the refusal of receiving treatment against medical advice in hospitalized patients in Iran Psychiatric Hospital.

Methods: The present study was a descriptive cross-sectional study. One hundred patients hospitalized in Iran Psychiatric Hospital discharged with personal consent against medical advice from July to December 2018 were studied. Two methods were used for assessment; the fulfillment of a routine ministry-approved checklist by the dischargers themselves and the face-to-face interview with both the patient and discharger based on a researcher-made checklist. Cohen's Kappa coefficient was used to assess the agreement of the answers of patients to both routine ministry-approved and researcher-made checklists by SPSS software version 16.0 with an overall accuracy of $95 \%$.
\end{abstract}

Results: Based on the results extracted from the researcher-made checklist, 43 (43\%) of the discharges were generally based on patient-related factors. The personal insistence to discharge by the patient was cited as the main reason for discharge. Cohen's Kappa coefficient showed no significant agreement between the patient's answers to the interview and what they have previously filled in the routine ministry-approved checklist. More specifically, the measure of agreement for answers of patients to questions in the standard checklist and the questions asked by the interviewer was $0.078(\mathrm{p}=0.167)$.

Conclusion: From the results of this study, it can be concluded that the face-to-face interview based on the researcher-made checklist can more effectively determine the reasons for discharge of patients due to the accuracy of the interview.

Keywords: Discharge against medical advice (DAMA), Psychiatric patient, Hospitalization

Conflicts of Interest: None declared

Funding: Iran University of Medical Sciences

*This work has been published under CC BY-NC-SA 1.0 license.

Copyright $\odot$ Iran University of Medical Sciences

Cite this article as: Soraya Sh, Zolfi M, Seddigh R, Mohammadsadeghi H, Hadi F. Discharge of psychiatric patients against psychiatrist's advice. Med J Islam Repub Iran. 2021 (19 Jul);35:92. https://doi.org/10.47176/mjiri.35.92

\section{Introduction}

Discharge against medical advice (DAMA) is a condition in which the patient intends to leave the hospital early despite medical advice indicating that the patient is dissat-

Corresponding author: Dr Mohaddese Zolfi, zolfi.m@iums.ac.ir

${ }^{1 .}$ School of Medicine, Iran University of Medical Sciences, Tehran, Iran

2. Spiritual Health Research Center, Iran University of Medical Sciences, Tehran, Iran isfied with the services provided or a significant problem (1). The critical tasks of the hospital are to ensure the health status of the pati ents and to improve the quality of

个What is "already known" in this topic:

Using the standard checklist to determine the reasons for discharge of psychiatric patients against psychiatrist's advice cannot correctly determine the main reasons for discharge due to the exceptional circumstances of patients when they wish to leave the hospital.

\section{$\rightarrow$ What this article adds:}

Completing the standard checklist based on face-to-face interviews with patients who wish to be discharged from the psychiatric ward against their psychiatrist's advice helps to determine the main reasons for discharge more correctly. The resulting information can also help by providing the chance to change the patient's decision for discharge against medical advice. 
services provided to them. Patient's satisfaction with the quality of services provided and medical and nursing care are essential indicators in evaluating the quality and effectiveness of the health system (2). Various issues may play a role in increasing DAMA, such as demographic factors, mental health status, comorbid physical illnesses, previous hospitalizations, hospital services dissatisfaction, financial problems, family problems, caregiver-patient communication, lack of significant improvement in hospital, belief in traditional medicine, discomfort with an extended stay in the hospital, feeling of recovery, and the place of living (urban or rural).

Patients admitted to psychiatric hospitals and departments are more likely to leave the hospital against medical advice than patients admitted to the internal and surgical departments. Evaluation of the factors associated with the discharge against medical advice seems necessary because the failure of patients to complete the course of treatment is a risk factor for relapse, readmission, and the imposition of additional costs for the patient (3). Also, examining the causes and factors related to discharge against medical advice, the weaknesses of mental health care, and the necessary steps for improvement could be identified (4). Due to the lack of sufficient studies on this issue in Iran and the necessity of knowing the factors related to DAMA in psychiatric patients, this study aimed to investigate the factors related to discharge against medical advice in psychiatric patients using two different methods, including 1) fulfillment of routine ministry-approved checklist by the dischargers themselves and, 2) interviewing with both the patients and dischargers based on the researcher-made checklist.

\section{Methods}

\section{Data collection}

The present study was a cross-sectional, descriptive, and analytical study performed on 100 patients who had been decided to discharge against their psychiatrist's advice from Iran Psychiatric Hospital from July to December 2018. In this regard, the routine checklist made by the Ministry of Health, Treatment, and Medical Training (MOHTME) and the interview based on a researchermade checklist was used. The routine checklist of discharge against medical advice (DAMA) is a proposed form that its validity and reliability have been evaluated and approved by the MOHTME and is a typical form that has to be fulfilled by the patients who want to leave the hospital against medical advice. A researcher-made checklist is a form that is compiled by a focused group of psychiatrists working at Iran University of Medical Sciences as faculty members. Several interviews were also conducted with the families of patients and the nursing staff of Iran Psychiatric Hospital to prepare a checklist. Despite the more significant number of factors mentioned in the researcher-made checklist, its difference with the routine ministry checklist was not significant. In other words, the difference between the two checklists is negligible.

One hundred patients who were discharged against medical advice during this study were interviewed. Most of the patients were interviewed in a face-to-face manner in the hospital on the day of discharge, and others were interviewed by telephone within one week of hospital discharge. The routine ministry-approved checklist and the researcher-made checklist were completed by interviewing patients and dischargers. Finally, the results of these two assessment methods were compared. The Ethical Committee of Iran university of medical science approved this study (Ethical code: IR.IUMS.FMD.REC.1398. 227). Besides, verbal consent from the participants was received, and it was assured that all information was confidential.

\section{Inter-rater reliability}

Cohen's Kappa coefficient of Agreement: In conceptual inferential statistics, there is a concept that is called a measure of agreement that examines and evaluates the relationship between two quantities (5). The difference between this concept and other statistical correlations is the separation of two quantities by two individuals, phenomena, or two sources of decision making (6). A factor measures the size of the agreement called the Cohen's Kappa coefficient (7). What Cohen's Kappa coefficient seeks is the magnitude of agreement between two individuals, phenomena, or sources of decision making.

Suppose the raters are in complete agreement when the Cohen's Kappa coefficient is equal to one. If there is no agreement among the raters other than what would be expected by chance, the Cohen's Kappa coefficient is zero. The negative value of Cohen's Kappa coefficient, which implies no effective agreement between the two raters, or the agreement is worse than random. In this study, Cohen's Kappa coefficient was used to determine the agreement between the interviews of respondents with questions of the researcher-made checklist and the checklist made by the Ministry of Health. This test was done by SPSS software version 16.0.

\section{Results}

Results of demographic information: The mean age of the patients (100 patients) was 31 , ranged from 17 to 63 ; 71 patients $(71 \%)$ were under 35 years old. In this study, 18 patients $(18 \%)$ admitted to the emergency ward, 3 patients $(3 \%)$ admitted to the female ward, $34(34 \%)$ of patients admitted to the Mehr ward, 31 patients $(31 \%)$ admitted to the male-one ward, 4 patients $(\%)$ admitted to the male-two ward, and 10 patients (10\%) admitted to the male-three ward were discharged against medical advice.

The results also show that $57 \%$ of the discharges were based on the person's own decision. While $15 \%$ of discharges were based on their companions, and $28 \%$ were based on both factors. However, $21 \%$ of patients under study had a previous history of discharge against medical advice. A summary of the results is listed in Table 1.

Results of Patient's hospital records: From July to December 2018, 1115 individuals were discharged from the Iran Psychiatric Hospital, that 148 persons (13.27\%) were discharged against medical advice. The average length of stay until DAMA in different departments was 9.5 days. More specifically, it was 4 days in the average length of stay for the emergency ward, 6.5 days in the female ward, 


\begin{tabular}{|c|c|c|c|}
\hline Variable & & Number & Percent (\%) \\
\hline \multirow[t]{2}{*}{ Age } & $<35$ years old & 71 & 71 \\
\hline & $>35$ years old & 29 & 29 \\
\hline \multirow{8}{*}{$\begin{array}{l}\text { The person responsible for the } \\
\text { discharge }\end{array}$} & Brother & 7 & 7 \\
\hline & Daughter & 1 & 1 \\
\hline & Father & 56 & 56 \\
\hline & Grandfather & 1 & 1 \\
\hline & Mother & 16 & 16 \\
\hline & Parents* & 2 & 2 \\
\hline & Sister & 1 & 1 \\
\hline & Wife & 16 & 16 \\
\hline \multirow{3}{*}{ Marital status } & Divorced & 4 & 4 \\
\hline & Married & 40 & 40 \\
\hline & Single & 56 & 56 \\
\hline \multirow{5}{*}{ Education } & Associate of science & 9 & 9 \\
\hline & Licentiate degree & 8 & 8 \\
\hline & Diploma & 52 & 52 \\
\hline & illiterate & 2 & 2 \\
\hline & Lower than diploma & 29 & 29 \\
\hline \multirow[t]{2}{*}{ Patient's place of residence } & Tehran city & 35 & 35 \\
\hline & Other areas & 65 & 65 \\
\hline
\end{tabular}

* At the same time, the mother and father were present and had requested discharge.

5.3 days in the Mehr ward, 14 days in the male-one ward, 15.1 days in the male-two ward, 12.4 days in and malethree ward. Also, the results of the interview showed that the next destination in $99 \%$ of the patients after DAMA was home.

Based on the results, 46 patients $(46 \%)$ had a history of drug use, and 35 patients (35\%) had previous alcohol use. Moreover, 57 patients $(57 \%)$ had a history of suicide attempts, and 25 patients $(25 \%)$ had a history of physical aggression. In 27 patients (27\%), relatives had a history of hospitalization at a psychiatric ward which 2 patients (2\%) were discharged against medical advice. Besides, 16 patients $(16 \%)$ discharged against medical advice had medical comorbidity, and 45 patients (45\%) suffered from personality disorders.

Regarding their principal psychiatric diagnosis, Major Depressive Disorder $(\mathrm{n}=28)$, followed by Bipolar Disorder type II $(n=14)$, cluster B personality disorder $(n=14)$, and Schizophrenia $(n=14)$, were the most diagnosed disorders in this population (Fig. 1).

Reasons for Discharge against medical advice: The reasons for discharge against medical advice in a researchermade checklist consisting of 28 items, based on the standard hospital checklist, were organized into five main factors: patient-related factors, staff-related factors, hospital environment-related factors, treatment-related factors, and hospital facilities- related factors.

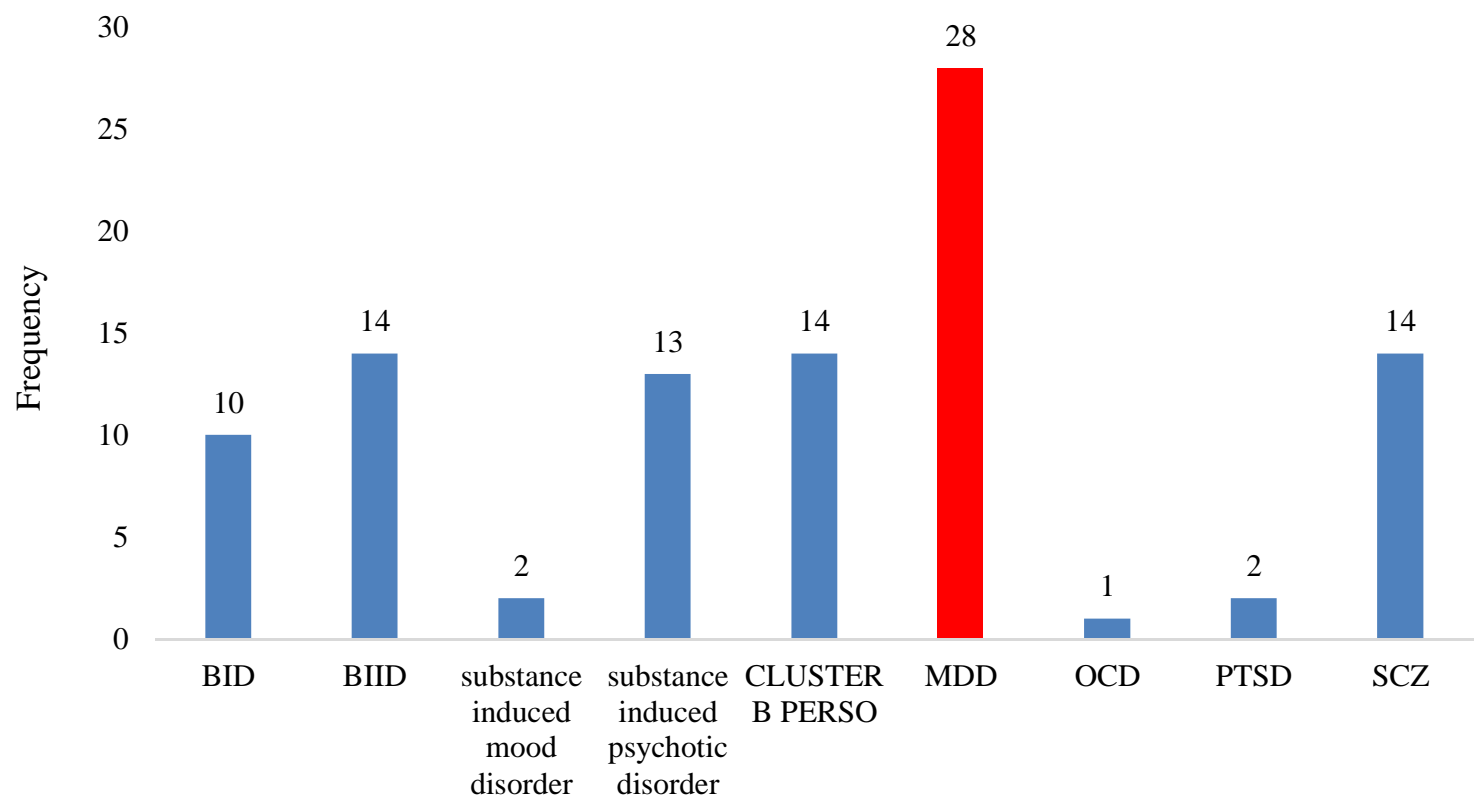

Fig. 1. The frequency of psychiatric illnesses in patients DAMA 


\section{(I) Patient-related factors}

According to our interviews, 43 discharges (43\%) were based on patient-related factors, with the highest frequency. Among the patient-related factors, the shares of personal insistence was detected in 45 patients, the shares of sense of improvement in 12 patients, the shares of job issues in 12 patients, the shares of feeling embarrassed by hospitalization in 8 patients, the shares of the necessity to attend ceremonies in 6 patients, the shares of need for companionship in the hospital in 2 patients, and the shares of family misconception about hospitalization was detected in 2 patients. None of the patients reported the expiration of insurance or financial support as a reason for discharge. It should be noted that each patient had the right to state different reasons.

\section{(II) Factors related to the hospital environment}

The results of the interviews in this study showed that $41 \%$ of discharges had reasons related to the hospital environment. Among the reasons, "to be worry about the negative impact of other patients" was a critical reason for discharge AMA (30 patients ). Also, high noise and inadequate relaxation (in 21 patients), the possibility of smoking (14 patients), lack of amenities (13 patients), and many hospitalized patients (12 patients) have listed as the common reasons for discharge against medical advice. In comparison, 10 patients and 2 patients were listed as "dissatisfied with nutrition" and "difficulties in reaching the hospital" for the reasons of discharge AMA.

\section{(III) Factors related to health care providers}

According to the results, 14 patients $(=14 \%)$ requested to discharge against medical advice based on factors associated with health care providers. "Inadequate nursing care" was the most important factor that 17 patients cited as one reason for DAMA. It should be noted that 9 patients complained about "medical care," and 9 patients complained about "improper communication". Of the other causes among the interviewees, only two percent left the hospital due to "restraint". Finally, only 2 patients left the hospital for treatment-related factors, such as feeling unhealthy. None of the patients "needed to be referred to other centers" did mention as reasons for DAMA.

Results of Cohen's Kappa coefficient of Agreement: As mentioned before, Cohen's Kappa coefficient was used to evaluate the agreement of the answers of patients to questions in the standard checklist and the questions asked by the interviewer. More specifically, the measure of agreement for answers of patients to questions in the standard checklist and the questions asked by the interviewer was calculated 0.078 (p-Value $=0.167$ ). Since the Cohen's Kappa coefficient was very close to zero and not significant at the $5 \%$ level, there is no significant agreement between the answers of patients to the standard checklist and the questions of interviewers.

\section{Discussion}

This study was a cross-sectional, descriptive, and analytical study to investigate the causes of DAMA based on the researcher-made checklist and the standard checklist.
The researcher-made checklist was completed through an open-ended interview with the patient and his or her companion. Interviews were done at the time of discharge or by telephone during the first week after discharge. The standard checklist was completed only by the patient's companion at the time of discharge; thus, the chance to interview and examine the reasons of patients for DAMA was one of the benefits of this study.

According to the data of the present study, 1115 individuals were discharged from Iran Psychiatric Hospital, 148 persons of whom were $(13.27 \%)$ discharged against medical advice. Other similar studies in the psychiatric ward reported DAMA ranging from 6 to $35 \%$. The average length of hospitalization was 9.5 days, while $88 \%$ of patients were hospitalized within 1 to 15 days. This finding is in line with Javad Setareh et al. (8) at Zare Hospital in Sari, Iran. Patients with psychiatric disorders are more likely to be discharged at an early stage due to issues related to the stigma of hospitalization in psychiatric wards. Besides, during the early days of hospitalization, the trust between patients and staff has not been fully established. Also, among those discharged against medical advice, the prevalence of those who experienced the first-time hospitalization was higher than those who had previous psychiatric hospitalization ( $60 \%$ vs. $40 \%$ ). This finding is in line with the study carried out by Sheikhmoonesi et al. (9) in 2011 at Zare Hospital in Sari. It may be argued that not acquainting patients and their families with the hospital environment may prevent them from receiving hospitalbased treatments.

Also, according to the results of interviews, the most frequent reason for DAMA was related to unreasonable patient insistence (45\%), concern about the negative impact of other patients (30\%), and high noise and lack of comfort in the department $(21 \%)$, respectively. None of our patients mentioned "costs of hospitalization", "expiry of insurance", and "need to be referred to other centers." In a cross-sectional study conducted by Javad Setareh et al. (8) at Zare Hospital in Sari, the most frequent reasons for DAMA were: "family insistence" (44\%), "patient insistence" (33\%), "dissatisfaction with treatment staff" $(12 \%)$, and "costs of hospitalization and family problems" $(12 \%)$. As these reasons were the reasons reported by the families of patients, one of the limitations of the above study was the lack of consideration of attitudes of patients.

The results of our study showed that among nearly half $(49 \%)$ of those who strongly insisted on DAMA, the frequencies of "substance use disorder" and "borderline personality disorder" were more than other causes related to DAMA. This finding is consistent with most similar studies, including the study of Javad Setareh et al. (8).

Based on the results of this study, it was concluded that there is a lack of insight and denial of the disorder by personality traits and temptation for substance abuse in patients with substance use disorder and patients with personality disorders. It has been hypothesized that the comorbidity of these two disorders with high levels of specific traits is the reason for DAMA. The reasons for DAMA as mentioned by most of the patients were "I am tired of the closed environment" or "I was bored in the 
closed environment".

Also, among the other factors related to DAMA were "concerns about the negative impact of other patients," as well as "high noise level and less relaxed environment", which were most frequent among the discharged patients admitted to the emergency ward. It seems that patients admitted to the emergency ward are in a more severe phase of the disorder and have more aggressive behaviors on arrival. This condition makes the situation more uncomfortable for other patients and may increase the rate of DAMA. Also, $37 \%$ of the families of discharged patients described the non-separated environment between male and female patients as one of the factors related to their DAMA.

The next factor in DAMA was "feeling recovered". To explain this, it should be noted that a lack of insight into the disorder reduces one's tolerance for hospitalization. Thus timely awareness and early psychoeducation are essential for the tolerance of hospital-based treatments. A "large number of hospitalized patients" and the "lack of amenities" (with similar percentages of $12 \%$ and 13\%) were other factors of DAMA. Suggestions made by several patients to improve their amenities included "to be a television in each room", "improved cooling and heating systems", and "increased walking time in the yard, outside the wards".

Although in the interview with patients, "feeling of recovery" accounted for $12 \%$ of causes of DAMA, data obtained from completing the hospital checklist indicated that "feeling recovery" accounts for $79 \%$ of DAMA. This incongruence can be explained by not probing the reasons for DAMA through fulfilling the routine checklist.

Finally, it can be said that due to the type and conditions of the interview, the use of the researcher-made checklist is more accurate than the standard checklist, showing the reasons for the patient's discharge with personal consent. Accordingly, it is recommended that in future studies and actions by taking into account the reasons for DAMA, the solution to existing problems be presented and studied. Then, the effects of these solutions on the decisions of patients for personal discharge were investigated.

One of the limitations of our study is its cross-sectional design and not comparing demographic factors and the type of clinical diagnosis of these patients with commonly discharged patients. In future studies, follow-up of patients who have been discharged against medical advice may be considered in terms of subsequent complications, including readmission.

\section{Conclusion}

The reduction of DAMA is so essential in psychiatric patients because failure to complete treatment is a risk factor for relapse, readmission, and additional costs for the patient. If physicians can initially identify patients at risk for DAMA, they can think of necessary measures to increase the acceptance of patients from hospitalization. From the results of this study, it can be concluded that the use of the researcher-made checklist to collect data can more clearly show the reasons for discharge of patients due to the accuracy of the interview and the patient's calmness to answer.

\section{Conflict of Interests}

The authors declare that they have no competing interests.

\section{References}

1. Spooner KK, Salemi JL, Salihu HM, Zoorob RJ. Discharge against medical advice in the United States, 2002-2011. In: Mayo Clinic Proceedings. Elsevier; 2017. p. 525-35.

2. Sodani PR, Sharma K. Assessing patient satisfaction for investigative services at public hospitals to improve quality of services. Nat J Com Med. 2011;2:404-8.

3. Vejdani M, Molavi Taleghani Y. Reasons for Discharge against Medical Advice among Patients Admitted to Ghaem ResearchTraining and Medical Center of Mashhad, Iran, in 2015. J Patient Saf Qual Improv. 2018;6(1):656-61.

4. Beckmann KM, Hume Z. An Allied Mental Health Aide Memoire to Disposition of Minors, Following Presentation to Emergency Department with Mental Health Difficulties. 2015.

5. Tang W, Hu J, Zhang H, Wu P, He H. Kappa coefficient: a popular measure of rater agreement. Shanghai Arch Psychiatry. 2015;27(1):62-7.

6. Legesse DT, Gemeda F. Comparison of Two Parasitological Tests and Post Mortem Examination to Detect Prevalence of Bovine Fasciolosis at Ambo Town Municipal Abattoir, West Shewa Zone, Ethiopia. Open Access Libr J. 2015;2(08):1.

7. Ucar E, Ozhan E. The Analysis of Firewall Policy Through Machine Learning and Data Mining. Wirel Pers Commun. 2017;96(2):2891909

8. Setareh J, Allameh Y, Mohseni Moalemkolaei N, Bagheri M. Prevalence of Discharge against Medical Advice in Psychiatric Patients and Associated Factors. J Maz Univ Med Sci. 2018;28(166):226-30.

9. Sheikhmoonesi F, Khademloo M, Pazhuheshgar S. Patients discharged against medical advice from a psychiatric hospital in Iran: a prospective study. Glob J Health Sci. 2014;6(3):213. 